nungsbildes unter den Abgeordneten im Ausschuss nicht mehr zuverlässig rekonstruiert werden kann. Es ist vorstellbar, dass das Fehlen von Protokollen sich schon dann bemerkbar machen wird, wenn aus tagespolitischen Gründen - wie es ja oft passiert - die Hintergründe und Grundlagen für eine Entscheidung des Bundestages zuverlässig verifiziert werden müssen.

Pilz' Buch ist kein Ersatz für eine Geschichte des Auswärtigen Ausschusses (so Pilz selbst, S. 25), sondern hat die heutige Rolle des Bundestages und seines Auswärtigen Ausschusses in der auswärtigen und internationalen Politik im Blick (S. 26). Mit großem Ertrag fließen in die Arbeit Ergebnisse aus Gesprächen mit Abgeordneten, Mitarbeitern der Fraktionen, der Bundestagsverwaltung (hier dem Ausschusssekretariat) und des Auswärtigen Amts ein. Zur Charakterisierung des Einflusses der Parlamentarier in der auswärtigen Politik streut Pilz gelegentlich Anekdoten ein, wie über Carlo Schmids Trinkfestigkeit bei dem Besuch Konrad Adenauers 1955 in Moskau oder den Bericht über Hans Sterckens Aufenthalt im Sudan 1989, als dieser in einer Direktübertragung im sudanesischen Fernsehen dem durch Putsch ins Amt gelangten Militärdiktator zurief: „Mr. President [...] don't kill your people.“ (S. 127 f.) Mit gleichem Kurzweil liest sich von gelegentlich geäußerten despektierlichen Vorurteilen, der Auswärtige Ausschuss sei aufgrund seines hohen Anteils an verdienten Altparlamentariern und ehemaligen Ministern ein „Altersruhesitz“ oder „Elefantenfriedhof". Pilz widerlegt auch die Behauptung von Marieluise Beck, der Auswärtige Ausschuss sei ein Ausschuss mit „viel Renommee und wenig Arbeit“ (S. 19), und bewertet seine Bedeutung im Vergleich mit dem Haushaltsausschuss oder dem Ausschuss für Wirtschaft und Arbeit, der immerhin ein zentrales gesellschaftspolitisches Feld zu bearbeiten hat. Er gesteht zu, dass der Haushaltsausschuss das „Königsrecht“ des Parlaments wahrnimmt und mit Geld Politik gemacht wird (S. 176), doch betont er, dass der Auswärtige Ausschuss die Entscheidungen des Bundestages über Krieg und Frieden vorbereitet. Erfreulich ist auch die handbuchartige Darstellung der Mitwirkung des Bundestages in interparlamentarischen Gremien wie in der IPU, der Parlamentarischen Versammlung des Europarates, der WEU, NATO und OSZE.

Pilz kann für sich mit dieser Arbeit in Anspruch nehmen, einen originären Beitrag zu einem bedeutenden Thema der Demokratie geleistet zu haben: der parlamentarischen Kontrolle der Außenpolitik.

Michael F. Feldkamp

\title{
Rituale in Parlamenten
}

Crewe, Emma und Marion G. Müller (Hrsg.): Rituals in Parliaments. Political, Anthropological and Historical Perspectives on Europe and the United States, Peter Lang Verlag, Frankfurt am Main u.a. 2006, 209 Seiten, € 39,-.

Der Frage, ob parlamentarische Rituale von Bedeutung sind, widmet sich der vorliegende Band, der auf englischsprachigen Beiträgen einer Konferenz im Warburg-Haus Hamburg basiert. Es ist ein explorativ angelegtes Buch, das nicht beabsichtigt, eine neue einheitliche Theorie oder einen eigenständigen Theoriebeitrag zu leisten, sondern das Potential für in- 
terdisziplinäre Studien auf diesem Feld illustrieren möchte. Dafür haben die Herausgeberinnen Emma Crewe und Marion G. Müller Politikwissenschaftler, Ethnologen und Historiker aus Frankreich, den USA, Ungarn, Großbritannien und Deutschland gewonnen, die entweder in der Wissenschaft oder in der Praxis, also den Parlamenten selbst, tätig sind. Neben einem einleitenden Beitrag stehen sechs Fallbeispiele und abschließend zwei eher konzeptionelle und theoriegeleitete Texte. Als Beispiele werden Zweite Kammern (britisches Oberhaus und US-Senat), nationalstaatliche Parlamente (Französische und Ungarische Nationalversammlung) sowie ein subnationalstaatliches (Schottisches Parlament) untersucht.

Die Hauptfrage, die das Thema des Buches sogleich aufwirft, lautet: Was sind überhaupt Rituale? Zunächst werden aber eher Beschreibungen geliefert, was Rituale leisten können. Eine Definition findet sich erst im letzten Beitrag, in dem Müller vorschlägt, parlamentarische Rituale seien Prozeduren, die für die Durchführung der parlamentarischen Geschäfte notwendig sind (S. 185). Damit wird beispielsweise auch die wöchentlich stattfindende Ausschusssitzung zum Ritual. Dies kann so definiert werden, ließe sich aber auch als Alltagsroutine bezeichnen. Hierin liegt eine Schwäche der Publikation: Auch wenn kein Theoriebeitrag angestrebt wird, wären definitorische Konkretisierungen angebracht, gerade auf einem Feld, das - so Crewe und Müller - noch zu wenig beachtet und untersucht wird. Zu klären wäre also, was ein Ritual ausmacht und wie es zum Beispiel gegenüber Routinen und Zeremonien abzugrenzen ist. Bilden letztere eine Untergruppe zu Ritualen, oder stehen sie gleichberechtigt neben ihnen? In welcher Beziehung stehen Routinen und Rituale zueinander? Kann eine Routine ab einem bestimmten Punkt (welchen?) zum Ritual werden? Zu fragen ist auch, ob Rituale Symbole sind, wie sie von Werner J. Patzelt in seinem Beitrag beschrieben werden. Auch die Affinität des Begriffs Ritual zum Religiösen sollte stärker diskutiert und ins Bewusstsein gebracht werden. Welcher Gewinn ist daraus zu ziehen, diese Begrifflichkeit auf inzwischen säkularisierte Institutionen, die aber zum Teil - das zeigt der Text über den US-Senat - die Nähe zur Religion suchen, anzuwenden?

Es scheint daher angebracht, Routinen, Rituale und Zeremonien begrifflich zu trennen und so zu schärfen. Eine Routinehandlung könnte in der wöchentlich oder zumindest regelmäßig stattfindenden Ausschusssitzung gesehen werden, ein Ritual in der (reglementierten) Ausschmückung einer Plenardebatte wie das Eintreten des Parlamentspräsidenten, die Kleidung, die Beiwerke wie der Hammer, und der Begriff der Zeremonie würde für außeralltägliche Veranstaltungen reserviert sein (wohingegen Routine und Rituale sich auf den Alltag bezögen) wie die Eröffnung eines Parlaments nach einer Wahl, die Vereidigung von Abgeordneten oder eine Gedenk-/Festveranstaltung des Parlaments. Legt man diese begrifflichen Annäherungen zugrunde, finden sich als Themen in den Beiträgen nicht nur Rituale, sondern auch Routinen, Zeremonien, die Sozialisierung von Abgeordneten sowie Geschichtspolitiken.

Marc Abélès interpretiert anhand von Beobachtungen der Französischen Nationalversammlung 1999 die Gesetzgebung als semiotischen Wettbewerb und rituellen Kampf, bei dem Menschen, die verschiedene Gesellschaftsteile verkörpern, miteinander konfrontiert werden. Er bezeichnet dies als guten Kampf und wendet sich dagegen, den Umgang der Abgeordneten untereinander als Theater oder Theatralisierung zu bezeichnen. Wenn es eine Show sei, dann eine, die auf sehr realen politischen Kräften beruhe. Auch Bernard Moreau untersucht das französische Parlament. Er widmet sich den militärischen Ritualen dort, die bereits seit 1800 existieren und heute noch zur Eröffnung jeder Nachmittagssitzung des 
Plenums exerziert werden. Sie dienen dazu, die Unabhängigkeit der Nationalversammlung fortlaufend zu behaupten. Das Militär, das eigentlich als Instrument der Exekutive agiert, gelangt hier in die Hände des Parlaments als Zeichen seiner Autonomie.

Nach László Kürti werden politische Rituale entwickelt, um politische Ideologie(n) zu dramatisieren. Ihn interessiert Ritualpolitik, also inwiefern Rituale entworfen, erfunden und angepasst werden können. Er macht an der Ungarischen Nationalversammlung deutlich, dass die Entstehung von Ritualen steuerbar ist: So schuf die dritte Regierung nach 1990 ein Zentrum für nationale Bilder, das intensiv die Berichterstattung über das Parlament lenkte, und sorgte dafür, dass die Heilige Krone aus dem Nationalmuseum in die zentrale Halle des Parlaments wechselte. Alastair J. Mann setzt sich mit einer wieder belebten Zeremonie auseinander, und zwar mit der Eröffnung des 1999 erneut geschaffenen Schottischen Parlaments, die als Prozession beziehungsweise Ritt an Vorgänger der bis 1707 existierenden Repräsentativkörperschaft anschloss, aber auch neue Akzente setzte. Die Grundstruktur wurde beibehalten (die Abgeordneten begeben sich zu Fuß zum Parlament, die Königin fährt vom Palast dorthin, und Insignien Schottlands werden zum Parlamentsgebäude transportiert), die Ausgestaltung jedoch modernisiert. So war die Eröffnung von 1999 musikalischer und säkularer als ihre Vorgänger, beteiligte mehr Frauen und Kinder sowie Parteien statt Stände und betonte den Anspruch eines multiethnischen und multireligiösen Schottlands.

Crewe und Richard Baker beschäftigen sich mit den Zweiten Kammern in Großbritannien und den USA. Beide verweisen auf die schnelle Sozialisationskraft, das hohe Alter und die Bedeutung bestimmter ritueller und zeremonieller Handlungen der zwei Institutionen. Dabei wird deutlich, dass das britische House of Lords viel stärker mit Reformanforderungen konfrontiert wird als der US-Senat. Ersteres ist zudem von dem Paradox gekennzeichnet, dass es im institutionellen Machtgefüge eine schwächere Position einnimmt, außerinstitutionell aber über reichlichen symbolischen Besitz (symbolic property) verfügt, da die Lords einen herausgehobenen sozialen Status in der britischen Gesellschaft genießen. Während die Zweite Kammer in Großbritannien aber gerade aufgrund zahlreicher altertümlicher Feierlichkeiten wie die Einführungszeremonie für neue Lords oder das jährliche State Opening mit der Rede der Königin anachronistisch wirkt ${ }^{1}$, gehen Geschichtlichkeit und Gegenwart des US-Senats eher Hand in Hand.

Patzelt führt kurz in seinen anderswo weiter ausgeführten theoretischen Ansatz der institutionellen Analyse und sein Konzept der symbolischen und instrumentellen Funktionen von Parlamenten ein. ${ }^{2}$ Er verweist insbesondere darauf, dass Symbole nichts Zusätzliches für Institutionen darstellen, sondern ihr Lebenselixier sind, ohne das sie nicht bestehen könnten. Gerade für den für Parlamente so wichtigen Repräsentationsglauben der Bevölkerung seien Symbole wahrscheinlich wichtiger als rationale Argumente allein. Besonders problematisch sei dafür die dialektische Beziehung von formalen und informalen Prozessen. Nur erstere sind offen für eine plausible Symbolisierung; viele im Informalen stattfindende

1 So erweckt das State Opening mit den in der Nähe der vorn und herausgehoben platzierten Königin versammelten Lords den Eindruck von Machtfülle von Monarch und Oberhaus, während Unterhaus und Premierminister, dessen Regierungsprogramm die Königin vorliest, weiter hinten positioniert sind.

2 Werner J. Patzelt (Hrsg.), Parlamente und ihre Symbolik, Wiesbaden 2001; ders. (Hrsg.), Parlamente und ihre Funktionen, Wiesbaden 2003. 
Prozesse können hingegen nicht überzeugend für die breitere Öffentlichkeit symbolisiert werden. Die Forschung über politische Rituale und Symbole muss zudem sinnvollerweise mit Themen der institutionellen Legitimität verbunden werden. Müller beschäftigt sich abschließend mit Abgeordneteneiden, die sie als liturgischen Akt definiert. Sie verweist mit dieser Begriffsprägung auf die Antike, in der die Liturgie als Form des öffentlichen Dienstes galt, und definiert parlamentarische Liturgien als zeremonielle Prozesse, die der Sichtbarkeit in der Öffentlichkeit und der Bedeutungsübermittlung dienen. Sie unterscheidet als Hauptfunktionen der Eide die zeremonielle Symbolisierung des Mandatsbeginns und den materiellen Test der Verfassungskonformität der Abgeordneten. Ferner können die Eide Müller zufolge danach differenziert werden, ob sie religiös oder weltlich und militärisch oder zivilbürgerlich geleistet werden.

Insgesamt besticht der Band durch seine vielfältigen Einblicke in Rituale, Zeremonien, aber auch Routinen in verschiedenen Parlamenten. Die Auswahl der Fallbeispiele ist gelungen, da nicht nur Nationalparlamente untersucht werden. Es finden sich interessante Details und bedenkenswerte Interpretationen und Gedanken. In dieser Hinsicht erfüllt die Publikation den selbst gesetzten Anspruch. Wer mehr definitorische Klarheit erhofft, wird hier aber nicht fündig. Die Ausgangsfrage nach der Bedeutung der Rituale wird unterschiedlich beantwortet; ein gewisses Maß an Wichtigkeit sprechen ihnen alle Beitragenden zu, am weitesten geht Patzelt. Ein Resümee, das die verschiedenen Zugänge, Interpretationen und empirischen Informationen zusammenbindet und diskutiert, fehlt leider. So bleibt Patzelts Mahnung, nicht bei interessanten Ergebnissen stehenzubleiben. Der Band leistet aber eine wichtige Basis für zukünftig hoffentlich noch folgende theoriegeleitete Vergleiche.

Franziska Höpcke

\section{Demokratische Repräsentation mit monarchischer Geschichte}

\section{Manow, Philip: Im Schatten des Königs. Die politische Anatomie demokratischer Repräsenta- tion, Suhrkamp Verlag, Frankfurt am Main 2008, 170 Seiten, € 10,-.}

Moderne Demokratien verzichten gemeinhin auf üppig repräsentative Ornamente und übermäßig erhabene Selbstdarstellungsrituale. Während sich in früheren Jahrhunderten Monarchien in Form großer Paläste, Schlösser und Hofzeremoniell in Szene setzten, kommen demokratisch verfasste Staaten weitgehend ohne ausladende Repräsentationsgesten aus. In repräsentativen Demokratien findet das Prinzip der Volksherrschaft seinen sichtbaren Ausdruck im gewählten und damit demokratisch direkt legitimierten Parlament. Dennoch haben sich bestimmte Herrschaftsmerkmale aus der Zeit überkommener Monarchien - so argumentiert Philip Manow - bis in unsere Tage gehalten, die auch in modernen Demokratien fortleben. Damit sind demokratische Staaten nicht „nach-metaphysisch“ (S. 12), sondern in vielen Bereichen dauerhaft durch tradierte „Erinnerungsspuren“ (S. 10) überschichtet. So bilden heutige Demokratien letztlich keine vollständig durchrationalisierte, jeglichen zeremoniellen Pomps entkleidete Regierungsform; vielmehr ist auch in ihnen ein historisch eingeschliffenes Sediment vorhanden, das Zeichen alter Ordnungen bis in die Gegenwart erkennen lässt. Dieses Erbe früherer Herrschaftsformen nimmt Manow zum 